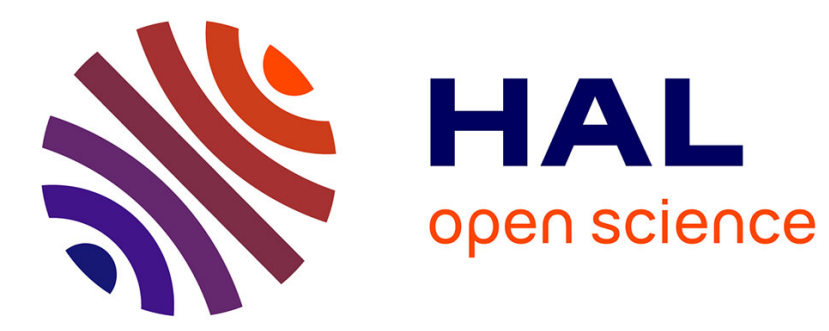

\title{
Evaluation of Tactile Sensors as an Alternative to Force Sensors in an Assistive Haptic Handlebar
}

\author{
A. Trujillo-Leon, F Vidal-Verdú, W. Bachta
}

\section{To cite this version:}

A. Trujillo-Leon, F Vidal-Verdú, W. Bachta. Evaluation of Tactile Sensors as an Alternative to Force Sensors in an Assistive Haptic Handlebar. 2017 IEEE Biomedical Circuits and Systems Conference (BioCAS), Oct 2017, Torino, Italy. pp.1-4, 10.1109/BIOCAS.2017.8325118 . hal-02497709

\section{HAL Id: hal-02497709 \\ https://hal.sorbonne-universite.fr/hal-02497709}

Submitted on 3 Mar 2020

HAL is a multi-disciplinary open access archive for the deposit and dissemination of scientific research documents, whether they are published or not. The documents may come from teaching and research institutions in France or abroad, or from public or private research centers.
L'archive ouverte pluridisciplinaire HAL, est destinée au dépôt et à la diffusion de documents scientifiques de niveau recherche, publiés ou non, émanant des établissements d'enseignement et de recherche français ou étrangers, des laboratoires publics ou privés. 


\section{Evaluation of Tactile Sensors as an Alternative to Force Sensors in an Assistive Haptic Handlebar}

\author{
A. Trujillo-León and F. Vidal-Verdú \\ Department of Electronics \\ Universidad de Málaga \\ Instituto de Investigación Biomédica de Málaga \\ Andalucía TECH \\ Málaga, Spain \\ Email: $\{$ atrujilloleon, fvidal\} @uma.es
}

\author{
W. Bachta \\ Agathe-Institut des Systèmes Intelligents et de Robotique \\ UPMC Université Paris 06 \\ CNRS UMR7222, INSERM U1150 \\ Paris, France \\ Email: wael.bachta@upmc.fr
}

\begin{abstract}
This paper shows experimental results that confirm that tactile sensors are appropriate to provide information about the caregiver intention in an assistive haptic handlebar. Preliminary tests have been performed to identify possible control inputs. Specifically, experiments have been carried out with a rear handlebar that incorporates tactile sensors and a force/torque sensor mounted on a powered wheelchair. An overall strong correlation is found between variables obtained from the tactile sensors output and those from the force/torque sensor that model the user interaction during driving. This way, an intuitive and easy driving can be achieved with a handlebar based exclusively on tactile sensors, which are a cost-effective alternative to force sensors.
\end{abstract}

\section{INTRODUCTION}

Developed societies must face the increasing aging of their inhabitants. In the coming decades, the predicted population pyramids for these countries will invert their shape [1]. In this context, research focused on the improvement of the elderly quality of life is more necessary than ever. Aging well requires health and functional capacity in the daily life activity [2]. This way, assistive technologies have to cope with one of the main age related issues: the mobility decline [3]. Canes and walkers are often prescribed for this purpose and, in recent years, robotic devices of this kind that assist the gait have been presented [4][5]. All of them are aimed at users with reduced mobility but still able to walk. However, there is a numerous group among the elderly with limited walking ability that requires other kind of mobility aid. In this sense, powered wheelchairs (PW) are reported as a means of extending the activity and participation of their users [6]. These chairs are normally driven through a joystick located at the end of the armrest. It is worth pointing out that this is not a suitable method for all users. Some of them, even those with experience, find it difficult and others may need the assistance of another person [7][8]. For others, it is almost impossible due to certain severe limitations: nervous system diseases, spinal cord injuries, blindness, mental disability, etc. When the user can not drive the PW properly by himself, the help of an assistant is required. Since propelling a wheelchair in day to day life is a hard task that may cause health problems [9], it is useful that assistants can benefit from PWs advantages. The common commercial driving solution aimed at assistants consists in another joystick placed at the rear part of the chair [10]. However, as has been reported, this device is not very comfortable and intuitive. Regarding research, authors of [11] present an assisting controller based on the forcevelocity relationship. It generates an assisting force when the attendant's propelling force exceeds a threshold. However, it does not contemplate steering and only simulations results are presented, so none sensor is proposed or evaluated to measure the input force. Kakimoto et al. propose a prototype of a power-assisted attendant propelled wheelchair that detects the caregiver propulsion force through a force sensor placed on the shaft of the handling bar [12]. Although the impulsion force is reduced by 50 to $60 \%$, the behavior is unstable under certain conditions. It was not intended to help in steering but in climbing ramps.

Assistive ambulatory devices incorporates often haptic interfaces since it makes them more intuitive and eases their handling. Most of them require force sensors, what increases considerably the cost and can prevent them from reaching the market. Tactile sensors could be an alternative to force sensors in haptic devices. Based on different principles of transduction (piezoresistive, capacitive, etc.), their main advantage is their cost-effectiveness. Moreover, they could also perform additional functions as user grip detection. In [13] we presented the implementation of a tactile handlebar aimed at PW assistants with the purpose of providing them with an easy and effective solution to drive a PW. A basic control algorithm was used to test the feasibility of the proposal. However, among other flaws, the user had to be instructed in order to use it, which reduced the intuitiveness. The operation of a regular handlebar, ability that most people have, can be expressed as a combination of force and torque that quantifies forward/backward and turns maneuvers, respectively. This work intends to identify which variables extracted from the tactile handlebar correspond with the force and torque exerted by the assistant so that the PW engines can be activated according to them. This way, the driving would be the same as that in a regular handlebar and, thus, very intuitive. An experiment was performed: ten participants grasped the handlebar attached to a PW and they drove it following a trajectory that included the most common maneuvers. A force sensor placed between the handlebar and the PW was used to extract ground-truth force and torque during the tests. The correlation between the signals captured by the force sensor and two parameters calculated from the pressure on the tactile sensors was studied. 
This paper is structured as follows. In Section II the hardware is described. The tactile handlebar, its electronics and the experimental setup are presented. Then, Section III explains the protocol followed to carry out the experiment and analyze the data. Section IV presents and discusses the results of the study. Finally, conclusions and future work lines are given in Section V.

\section{MATERIALS}

Firstly, the tactile handlebar is described. Then, the experimental setup designed to its study is detailed.

\section{A. TACTILE HANDLEBAR}

Designed to be grasped as a regular one, it has two handles, one for each hand, covered by rectangular commercial force sensing resistor sensors. The sensors are arranged forming a matrix of two by eight tactels. This organization aims to minimize the addressing resources needed to scan the arrays. The rows and columns of the matrix are wired to a custom conditioning electronics board based on a PIC18F4680 microcontroller. Thus, the reading procedure is as follows: a set of analog switches activates the row to be read. Then, the resistance variation in each tactel of the selected row is turned into a voltage variation by transimpedance amplifiers.

Finally, the amplifiers output is digitized by the analogto-digital converters that the PIC18F4680 incorporates. The microcontroller can send the pressure data to a computer via USB. The device together with its electronics costs less than $200 €$. Further details can be found in our work described in [13].

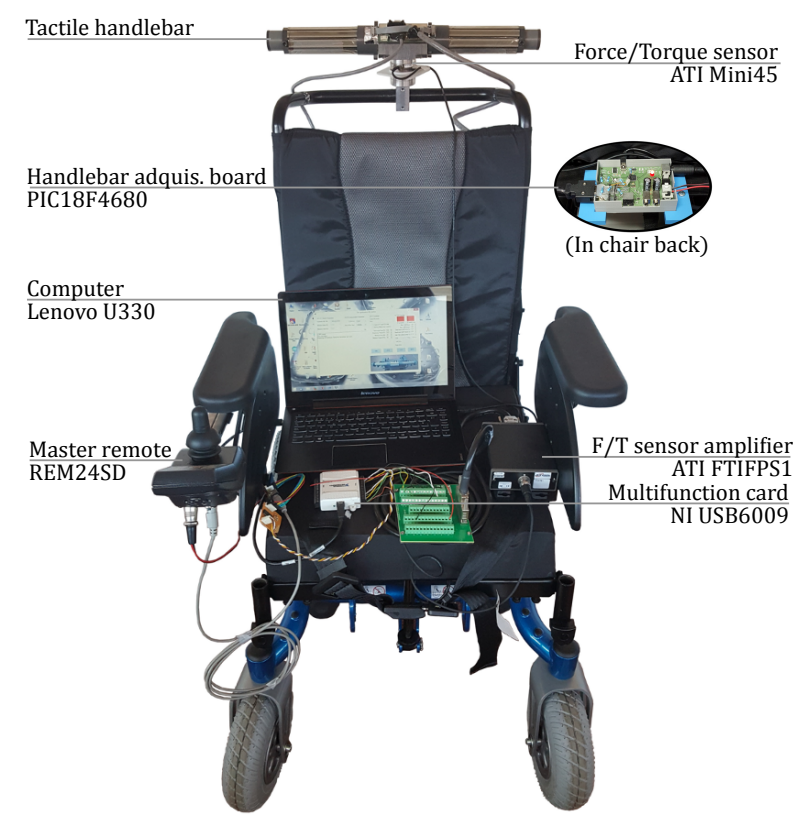

Fig. 1. Experimental setup.

\section{B. EXPERIMENTAL SETUP}

Figure 1 shows the experimental setup. It should be said that, although nobody is seated on the chair during the tests, the weight of the setup (PW frame, seat, batteries, etc.) is approximately the same of a conventional wheelchair with a $80 \mathrm{~kg}$ person on it. Moreover, since in the experiment an assistive driving system is emulated, the participant will not have to propel manually the heavy setup but the driving is assisted by the PW engines as it will be explained later. In the introduction it was said that the user interaction can be expressed in terms of the force and the torque exerted on the joint between the handlebar and the chair back. For this reason, an ATI Mini45 six axis force/torque sensor has been placed in this point, between the handlebar and the chair frame. This device is widely used in robotics (with a cost of several thousands of euros). It measures the force $\left(F_{y}\right)$ in the axis aligned with wheelchair direction of movement, i.e., the force exerted by the user when pushing or pulling the handlebar. In addition, it obtains the torque $\left(T_{z}\right)$ in the vertical axis, which is perpendicular to the walking direction. Note that this is the torque applied by the assistant during the turns. These two signals are conditioned by an ATI FTIFPS 1 amplifier and, afterwards, captured through the analog inputs of the multifunction card NI USB6009. Figure 2 can help to understand this operation.

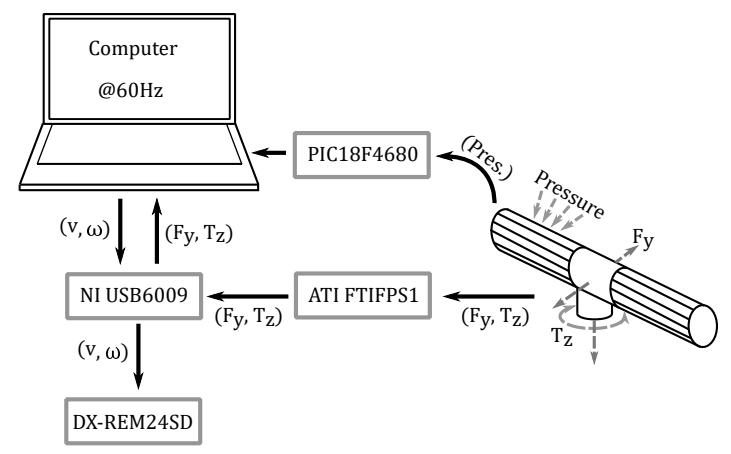

Fig. 2. Experimental setup scheme.

A computer is in charge of gathering synchronously the pressure and the force/torque information from the tactile handlebar and the ATI Mini45 sensor, respectively, at a rate of $60 \mathrm{~Hz}$. After every reading, the data are stored for further study. The control signals for the wheelchair engines are also obtained from the processing of these data. This is done by a personal computer plus an acquisition card that provides analog signals similar to those generated by a joystick to the master remote mounted on the chair, a DX-REM24SD. This remote provides the chair power module with two signals that represent the linear and angular desirable speeds. The linear speed, $v$, will be proportional to the force exerted on the handlebar when the user pushes or pulls, $F_{y}$. In the same manner, the angular speed, $\omega$, will vary proportionally with the torque measured on the handlebar when the user tries to turn, $T_{z}$. This dependance can be seen in (1).

$$
\begin{gathered}
v=G_{\text {lin }} F_{y} \\
\omega=G_{\text {ang }} T_{z}
\end{gathered}
$$

Where $G_{l i n}$ and $G_{a n g}$ may be used to tune the sensibility according to the assistant preferences.

The computer calculates $v$ and $\omega$, which are made into voltage signals by using the NI USB6009 analog outputs. The outputs of the NI USB6009 are connected to the master remote so that, 
after every sensors reading, it updates the linear and angular speed and activates the wheel engines accordingly. This way, the wheelchair moves following the user intention.

\section{METHODS}

In this section, the experimental protocol is explained. Later, the data processing is described.

\section{A. PROTOCOL}

Ten volunteers (P1-P10) without previous experience participated in the test. They were between 24 and 63 years old, with an average age of 39,6 . Given writing consent and complying with the ethical principles of the declaration of Helsinki at all times, they were asked to drive the wheelchair using the handlebar along a specified path. In order to help the participants to follow the trajectory, marks on the ground and plastic cones were used. The path gathers, for about 25 meters, the typical maneuvers present in the normal usage of a handlebar. These are: several forward movements, two $90^{\circ}$ turns, an open turn, a $180^{\circ}$ turn around and a backward movement. Around one minute is required to cover the path length. It is worth noting that the participants were not aware of the experiment purpose. Two trials were carried out. The first one was used to familiarize the participants with the system. During the second test $F_{y}, T_{z}$ and the tactile output were registered as explained in the previous point.

\section{B. DATA ANALYSIS}

As said before, this work intends to find parameters extracted from the tactile handlebar that allow an intuitive control of the wheelchair. The pressure data can be processed in several ways. However, not all methods meet our requirements. One of them has to do with the real-time performance. A variable easy to calculate allows simpler electronics, which maintains the cost-effectiveness of the proposal. Other valuable point is using a relative parameter. Whereas absolute variables suppose the direct reflection of the pressure on the handlebar, a relative variable obtained from the latter is more appropriate to detect patterns and subtle variations and it is more robust to the change of an user for other. Finally, the chosen parameter has to represent as well as possible the user intention as it will be shown in Section IV. The center of mass (CoM) (also known as center of pressure) satisfies these premises. It is a common way to summarize the pressure information on a surface composed of a group of tactels when there is an interaction with an object. It concentrates the data from all the handle elements in a single spatial coordinate that gives us information about how the pressure distribution is (Figure 3). In addition, it is not affected by pressure offset changes. This means that if the value of all the tactels increases or decreases the same, the $C o M$ does not vary. It can be obtained for the left and right handle with the expression shown in (2).

$$
C o M_{L / R}=\frac{\sum_{i=1}^{8} p(i) x(i)}{\sum_{1}^{8} p(i)}
$$

Where $x(i)$ and $p(i)$ are the position and the pressure value of the $i^{\text {th }}$ tactel in the handle for which CoM is being calculated.

$C_{L} M_{L}$ and $C_{R} M_{R}$ are computed for each trial. The initial and the final captured samples in which both centers of mass

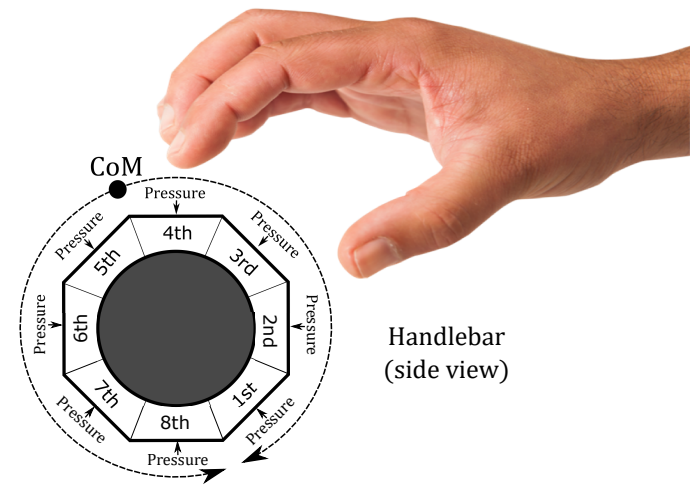

Fig. 3. Center of mass range of movement on the tactile array.

are not stable are discarded. These correspond to the very first moment in which the participant grasps the handlebar and to the instant when the user releases it at the end of test and they do not contain valuable information. From the latter parameters two variables are calculated. One of them is the sum of the centers of mass of each handle and the other their subtraction. Both can be seen in (3).

$$
\begin{aligned}
S U M_{C o M}(t) & =\operatorname{CoM}_{L}(t)+\operatorname{CoM}_{R}(t) \\
S U B_{C o M}(t) & =\operatorname{CoM}_{L}(t)-\operatorname{CoM}_{R}(t)
\end{aligned}
$$

Finally, the feasibility of these two variables as control inputs to process the wheelchair movement is studied. They are compared with the pair used in the experimental setup for this function, $F_{y}$ and $T_{z}$. This way, the correlation between the couples $\left\langle S U M_{C o M}, F_{y}>\right.$ and $\left\langle S U B_{C o M}, T_{z}>\right.$ is computed.

\section{RESULTS}

All the parameters used in the analysis have been previously low-pass filtered to remove possible noise and interferences. In order to assess the degree of coupling between the parameters, Pearson correlation was used. The results for $\left\langle S U B_{C o M}, T_{z}>\right.$ are: $r=0.95[\mathrm{P} 1], 0.84[\mathrm{P} 2], 0.85[\mathrm{P} 3]$, 0.88[P4], 0.70[P5], 0.80[P6], 0.68[P7], 0.67[P8], 0.84[P9], $0.82[\mathrm{P} 10]$ (all with $p<0.001$ ). For $\left\langle S U M_{C o M}, F_{y}>\right.$, they are: $r=0.91[\mathrm{P} 1$ ], 0.83[P2], 0.77[P3], 0.89[P4],0.71[P5], 0.83[P6], 0.56[P7], 0.80[P8], 0.78[P9],0.85[P10] ( $p<0.001$ for all of them). For $\left\langle S U M_{C o M}, F_{y}\right\rangle$, the correlation was high positive for nine participants and moderate positive for one of them. Regarding $<S U B_{C o M}, T_{z}>$, it was practically high positive for all the participants [14]. Moreover, it was statistically significant for all the tests in both cases.
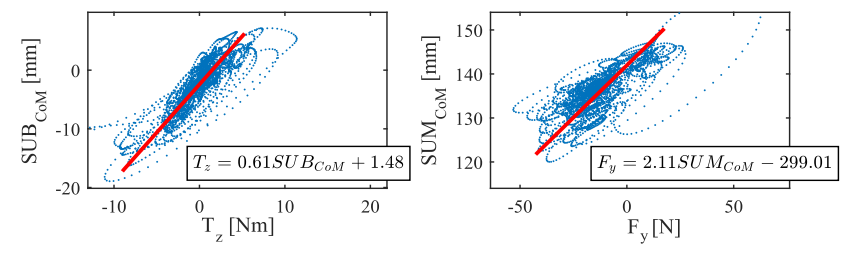

Fig. 4. Link between the assessed couples for an average participant.

This overall strong correlation implies a linear relationship between the involved variables that can be clearly observed 
in Figure 4. It depicts the two assessed couples with their corresponding $1^{\text {st }}$ order functions for one of the participants that had an average performance in the experiment, i.e., it was neither the best nor the worst. Although the results are generally solid, the participant $\mathrm{P} 7$ presents a $<S U M_{C o M}, F_{y}>$ correlation that, despite not being weak, is a little lower than the others. In this case, it seems that the first order function that fits in the test carried out by P7 deviates slightly from the data captured while pulling the handlebar (see Figure 5), which could affect to the linear correlation. This effect has not been observed in the rest of the tests.

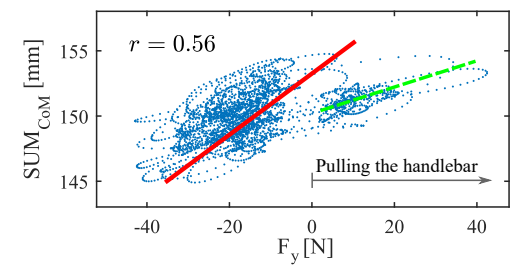

Fig. 5. SU $M_{C o M}$ versus $F_{y}$ for P7. The red line is computed from the data of P7 by linear regression. The green dashed line fits better with the data captured during pulling maneuvers.

A wide range of parameters, that are being studied at the time this work is written, could influence the correlation: the fact of comparing outputs from sensors of different kind, the hand size, the grip force or the way the user grasps the handlebar, among others. Although a deeper study may improve the correlation, the presented results are good enough to show that $S U M_{C o M}$ detects and quantifies push and pull maneuvers and $S U B_{C o M}$ does the same with turns. Apart from the presented parameters, others were also tested without success (derivative and integral of $C o M$ and their different combinations, absolute pressure values, etc.). Only left and right grip force subtraction, calculating the grip forces as the mean pressure on each handle, correlates well with $T_{z}$ in turn maneuvers. However, this situation is only valid when the PW is stopped and turning around itself $(v=0, \omega \neq 0)$. If the $\mathrm{PW}$ is moving forward/backward and turning at the same time $(v \neq 0, \omega \neq 0)$, the correlation becomes really low and it is no longer a viable parameter.

\section{CONCLUSIONS AND FUTURE WORK}

This paper shows the feasibility of tactile sensors as an alternative to costly force sensors in ambulatory haptic devices, specifically in a haptic rear handlebar, as a means to achieve an intuitive and easy driving. An experiment has been undertaken to collect and study the pressure on the tactile handlebar as well as the force registered by the force/torque sensor while driving. The data obtained for ten participants show a tight coupling between the parameters extracted from the force/torque sensor, $F_{y}$ and $T_{z}$, and others computed using the pressure on the tactile sensors, $S U M_{C o M}$ and $S U B_{C o M}$. This suggests that the tactile sensor could substitute the force sensor as input device in the control of the wheelchair. This point is relevant in terms of market realistic possibilities. Although the results are promising, it would be appropriate to carry out a formal controlled experiment to better assess performances. It should involve specific navigation measurements such as the average speed, the level of corrective measures during driving to stay on path, etc., as well as subjective factors as the perceived ease of use for the participants when using the different interfaces. This is an ongoing task by the time this work is presented. Future work would also involve the improvement of the control algorithm based on the tactile sensor output. Regarding forthcoming realizations, the designed electronics could be better exploited performing the whole control task, which avoid using a computer as it is done in this work.

\section{ACKNOWLEDGMENT}

This work was partially funded by the Spanish Government under contract TEC2015-67642-R, FPU Program and European ERDF funds. It has also been partly accomplished within the laboratory of excellence SMART supported by French state funds managed by the ANR within the Investissements d'Avenir programme under reference ANR-11-IDEX-0004-02.

\section{REFERENCES}

[1] "World Population Prospects: The 2015 Revision," United Nations. Department of Economic and Social Affairs, Population Division, 2015, URL: https://esa.un.org/unpd/wpp/publications/files/key_findings_wpp_ 2015.pdf [accessed: April, 2017].

[2] B. A. Hawkins, "Aging well: Toward a way of life for all people," Preventing Chronic Disease, vol. 2, no. 3, p. A03, July 2005.

[3] M. Rantakokko, M. Mänty, and T. Rantanen, "Mobility decline in old age," Exercise and Sport Sciences Reviews, vol. 41, no. 1, pp. 19-25, 2013.

[4] R. Ady, W. Bachta, and P. Bidaud, "Development and control of a onewheel telescopic active cane," in 5th IEEE RAS/EMBS International Conference on Biomedical Robotics and Biomechatronics, August 2014, pp. 461-466.

[5] V. Pasqui, L. Saint-Bauzel, C. Zonga et al., "Projet MIRAS : Multimodal interactive robot for assistance in strolling," Technologies pour la santé et l'autonomie, vol. 33, no. 2, p. 165-172, April 2012.

[6] A. Brandt, S. Iwarsson, and A. Ståhle, "Older people's use of powered wheelchairs for activity and participation," Journal of Rehabilitation Medicine, vol. 36, no. 2, pp. 70-77, March 2004.

[7] C. Torkia, D. Reid, N. Korner-Bitensky, D. Kairy, P. W. Rushton, L. Demers, and P. S. Archambault, "Power wheelchair driving challenges in the community: a users' perspective," Disability and Rehabilitation: Assistive Technology, vol. 10, no. 3, pp. 211-215, 2015. [Online]. Available: http://dx.doi.org/10.3109/17483107.2014.898159

[8] L. Fehr, W. Langbein, and S. Skaar, "Adequacy of power wheelchair control interfaces for persons with severe disabilities: a clinical survey," Journal of Rehabilitation Research and Development, vol. 37, no. 3, pp. 353-360, May/June 2000.

[9] J. Roberts, H. Young, K. Andrew, A. McAlpine, and J. Hogg, "The needs of carers who push wheelchairs," Journal of Integrated Care, vol. 20, no. 1, pp. 23-34, 2012. [Online]. Available: http://dx.doi.org/10.1108/14769011211202265

[10] "Dynamic Controls. DX2 Compact Attendant Remote Module," 2017, URL: https://dynamiccontrols.com/en/mobility-product-users/products/ $\mathrm{dx} 2 /$ secondary-remotes [accessed: January, 2017].

[11] T. Suzuki, H. Uchiyama, C. Holloway, and N. Tyler, "Assisting control for attendant propelled wheelchair based on force velocity relationship," in 2012 Annual International Conference of the IEEE Engineering in Medicine and Biology Society, Aug 2012, pp. 3073-3076.

[12] A. Kakimoto, H. Matsuda, and Y. Sekiguchi, "Development of powerassisted attendant-propelled wheelchair," in Engineering in Medicine and Biology Society, 1997. Proceedings of the 19th Annual International Conference of the IEEE, vol. 4, Oct 1997, pp. 1875-1876 vol.4.

[13] A. Trujillo-León and F. Vidal-Verdú, "Driving interface based on tactile sensors for electric wheelchairs or trolleys," Sensors, vol. 14, no. 2, pp. 2644-2662, February 2014

[14] D. E. Hinkle, W. Wiersma, and S. G. Jurs, Applied Statistics for the Behavioral Sciences, 5th ed. Houghton Mifflin, 2002. 